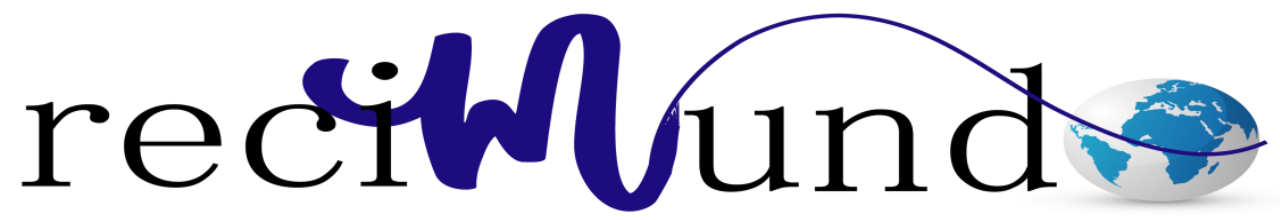

Revista Científica Mundo de la Investigación y el Conocimiento

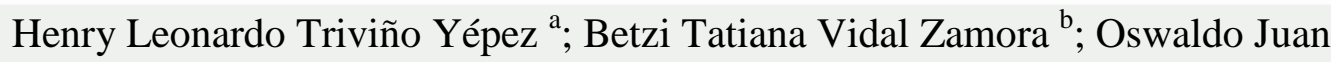
Zambrano Chávez ${ }^{\mathrm{c}}$

Cáncer colorrectal en el hospital Guayaquil Dr. Abel Gilbert Pontón en el 2016

Revista Científica Mundo de la Investigación y el Conocimiento. Vol. 2 núm., especial, mayo, ISSN: 2588-073X, 2018, pp. 734-755

DOI: 10.26820/recimundo/2.esp.2018.734-755

Editorial Saberes del Conocimiento

Recibido: 05/12/2017

Aceptado: $15 / 02 / 2018$
a. henry triye@ hotmail.com
b. betzividal.24@gmail.com
c. dr.oswaldozch@hotmail.com 


\section{Cáncer colorrectal en el hospital Guayaquil Dr. Abel Gilbert Pontón en el}

Vol. 2, núm. Esp., (2018)

Henry Leonardo Triviño Yépez; Betzi Tatiana Vidal Zamora; Oswaldo Juan Zambrano Chávez

\section{RESUMEN}

Introducción: La tasa de mortalidad de este tipo de cáncer se ha duplicado de 1997 a 2014. Un informe del hospital de Solca, en Guayaquil, lo ubica en el quinto lugar en mortalidad. $\mathrm{Su}$ incidencia también aumenta a medida que las familias tienen mayor acceso a consumir carnes rojas y procesadas. Materiales y métodos: La muestra fue de 109 pacientes que presentaron Cáncer Colorrectal incluidos 41 nuevos casos del Hospital Guayaquil en el período de tiempo de estudio establecido, se trata de un estudio de corte transversal, retrospectivo, descriptivo y observacional. Resultados: La mayor incidencia de casos de Cáncer Colorrectal se diagnosticó por encima de los 56 años de edad, Se determinó que entre 109 pacientes que fueron tratados e ingresados en el Hospital Abel Gilbert Pontón en el 2016, 41 fueron diagnosticados durante este mismo año, correspondiendo al 37,6\% de la muestra, El tipo de tumor más frecuente fue el ADENOCARCINOMA COLORRECTAL. Se Observó una mayor incidencia de Cáncer Colorrectal en pacientes masculinos con el $76,1 \%$ del total.

Palabras claves: Cáncer colorrectal, adenocarcinoma, linfoma, hemorragia digestiva baja, anemia. 


\title{
Cáncer colorrectal en el hospital Guayaquil Dr. Abel Gilbert Pontón en el 2016
}

Vol. 2, núm. Esp., (2018)

Henry Leonardo Triviño Yépez; Betzi Tatiana Vidal Zamora; Oswaldo Juan Zambrano Chávez

\begin{abstract}
Introduction: The mortality rate of this type of cancer has doubled from 1997 to 2014. A report from the Solca Hospital in Guayaquil places it in the fifth place in mortality. Its incidence also increases as families have greater access to consuming red and processed meats. Materials and methods: The sample was of 109 patients that presented Colorectal Cancer including 41 new cases of the Guayaquil Hospital in the period of time of established study, it is a transversal, retrospective, descriptive and observational study. Results: The highest incidence of Colorectal cancer cases was diagnosed over 56 years of age. It was determined that among 109 patients who were treated and admitted to the Abel Gilbert Pontón Hospital in 2016, 41 were diagnosed during this same year, corresponding to $37.6 \%$ of the sample. The most frequent tumor type was COLORECTAL ADENOCARCINOMA. A higher incidence of colorectal cancer was observed in male patients with $76.1 \%$ of the total.
\end{abstract}

Keywords: Colorectal cancer, adenocarcinoma, lymphoma, low digestive hemorrhage, anemia. 


\section{Cáncer colorrectal en el hospital Guayaquil Dr. Abel Gilbert Pontón en el}

Vol. 2, núm. Esp., (2018)

Henry Leonardo Triviño Yépez; Betzi Tatiana Vidal Zamora; Oswaldo Juan Zambrano Chávez

\section{Introducción.}

Este proyecto de investigación se encamina a la Línea de Investigación 4.1 del Ministerio de Salud Pública de la República del Ecuador con la Sublínea de Investigación sobre Perfil Epidemiológico. En cuanto al marco investigativo de la Universidad de Guayaquil nos enfocaremos a la Línea de Investigación de Salud Humana y Animal, cuya Sublínea es la de Investigación de Biomedicina y Epidemiología. Por último la Línea de Investigación del Área 5 y 8 y la Sublínea de Investigación 3 (Neoplasias Prevalentes) corresponden de manera local a la Facultad de Ciencias Médicas de la Universidad de Guayaquil. El cáncer colorrectal (CCR) es una enfermedad frecuente y letal. El riesgo de desarrollar CCR está influenciado por factores ambientales y genéticos; las tasas de incidencia y mortalidad de CCR varían notablemente en todo el mundo. A nivel mundial, el CCR es el tercer cáncer más comúnmente diagnosticado en los hombres y la segunda en las mujeres, con 1,4 millones de nuevos casos y casi 694.000 muertes estimadas que se han producido en el año 2012. (Boardman, 2011)

A nivel mundial, la incidencia de CCR varía a lo largo de su estudio. En los últimos datos de 2012 del Globocan, las mayores tasas de incidencia están en Australia y Nueva Zelanda, Europa y América del Norte, y las más bajas se encuentran en África, Asia del Sur y Centroamérica. Estas diferencias geográficas parecen ser atribuibles a diferencias en las exposiciones alimentarias y ambientales que se imponen a un fondo de la susceptibilidad determinada genéticamente. (Rodriguez-Bigas, 2016)

La edad es un factor de riesgo importante para el CCR esporádico. El cáncer de intestino grueso es raro antes de los 40 años; la incidencia comienza a aumentar de manera significativa 


\section{Cáncer colorrectal en el hospital Guayaquil Dr. Abel Gilbert Pontón en el 2016}

Vol. 2, núm. Esp., (2018)

Henry Leonardo Triviño Yépez; Betzi Tatiana Vidal Zamora; Oswaldo Juan Zambrano Chávez

entre las edades de 40 y 50, y las tasas de incidencia específicas por edad aumenta en cada década subsiguiente a partir de entonces.

Según cifras oficiales de la Sociedad de Lucha contra el Cáncer (SOLCA), aproximadamente 1.200 nuevos casos de cáncer de colon se presentan en el Ecuador cada año. De estos casos, si son tratados oportunamente, el $52 \%$ de personas sobreviven. La tasa de mortalidad de este tipo de cáncer se ha duplicado de 1997 a 2014. Un informe del hospital de Solca, en Guayaquil, lo ubica en el quinto lugar en mortalidad. Su incidencia también aumenta a medida que las familias tienen mayor acceso a consumir carnes rojas y procesadas. (SOLCA, 2010) El propósito de esta investigación es determinar y establecer la prevalencia y la presencia de nuevos casos durante el año 2016, este caso la incidencia del Cáncer Colorrectal en pacientes atendidos en el Hospital Abel Gilbert Pontón en las áreas de hospitalización clínica, Gastroenterología, Oncología y Emergencia, se realizará un estudio restrospectivo analítico y observacional de pacientes con Cáncer Colorrectal, sus factores de riesgo, sus principales manifestaciones clínicas, diagnóstico por colonoscopia y reportes del servicio de Oncología a la presentación de este problema de salud pública.

El objetivo de la presente investigación es determinar la prevalencia de Cáncer Colorrectal, sus manifestaciones clínicas y factores de riesgo en el Hospital Guayaquil Dr. Abel Gilbert Pontón durante el 2016.

\section{Metodología.}

Hospital de especialidades Guayaquil Dr. Abel Gilbert Pontón 


\section{Cáncer colorrectal en el hospital Guayaquil Dr. Abel Gilbert Pontón en el}

Vol. 2, núm. Esp., (2018)

Henry Leonardo Triviño Yépez; Betzi Tatiana Vidal Zamora; Oswaldo Juan Zambrano Chávez Área de cirugía y gastroenterología

Universo

El universo está conformado por todos los pacientes que ingresaron al Hospital Abel Gilbert Pontón con diagnóstico de cáncer colorrectal durante el año 2016. La muestra estará conformada por las pacientes que cumplan los criterios de inclusión.

\section{Viabilidad}

Este trabajo de investigación es un estudio viable de ser realizado, porque en el Hospital Dr. Abel Gilbert Pontón, el autor labora como Interno de Medicina de la Universidad de Guayaquil y se cuenta con la aprobación del departamento de Docencia e Investigación del Hospital que aprobó el estudio y permite el acceso a las historias clínicas.

Además, se cuenta con el apoyo de las autoridades de la Universidad de Guayaquil; el Dr. Carlos Andrés León Borja Tutor de Tesis y Docente de la Rotación de Medicina Interna en el Internado Rotativo 2016 - 2017.

Tipo de investigación

Descriptiva, Analítica, Retrospectiva, Observacional y Transversal.

\section{Consideraciones bioéticas}

Debido a que se trata de un estudio retrospectivo y en cumplimiento de los estándares de Bioética relacionados a los principios de: autonomía, beneficencia, confidencialidad y justicia, se 


\section{Cáncer colorrectal en el hospital Guayaquil Dr. Abel Gilbert Pontón en el 2016}

Vol. 2, núm. Esp., (2018)

Henry Leonardo Triviño Yépez; Betzi Tatiana Vidal Zamora; Oswaldo Juan Zambrano Chávez

recurrió al Departamento de Docencia e Investigación del Hospital Abel Gilbert Pontón para la respectiva aprobación del estudio. Además, se respetó la integridad de las pacientes asegurando la confidencialidad de toda la información personal recabada de las historias clínicas.

Instrumentos de evaluación o recolección de la data

La identificación de pacientes con diagnóstico de Cáncer Colorrectal ingresados en el Hospital Abel Gilbert Pontón de Guayaquil en el periodo de estudio, comprendido en el año 2016, se realiza a partir de las historias clínicas proporcionadas por el departamento de estadística, la información requerida se obtuvo de la revisión de las historias clínicas de los pacientes que cumplieron con los criterios de inclusión, se recolectaron los datos en una hoja de recolección de datos elaborada por el investigador y con la información recabada se conformó una base de datos en Microsoft Excel y el programa IBM - SPSS para la elaboración de tablas y gráficos de barras donde se representen las variables del estudio.

\section{Resultados.}

Las historias clínicas fueron proporcionadas por el departamento de estadística del Hospital Guayaquil Dr. Abel Gilbert Pontón, y esta incluyó datos de filiación de los pacientes como numero de historia clínica, genero, nombre, edad, antecedentes patológicos personales clínicos, quirúrgicos.

Luego se hizo la revisión de cada historia clínica, analizando si los pacientes atendidos cumplían los criterios de inclusión, se obtuvo más datos utilizados como variables intervinientes de tipo cualitativas y ordinales; datos como diagnóstico definitivo de Cáncer Colorrectal según 


\section{Cáncer colorrectal en el hospital Guayaquil Dr. Abel Gilbert Pontón en el}

Vol. 2, núm. Esp., (2018)

Henry Leonardo Triviño Yépez; Betzi Tatiana Vidal Zamora; Oswaldo Juan Zambrano Chávez

CIE 10, el tipo histológico, estadio según TNM, localización por endoscopia digestiva baja, datos de laboratorio y su respectivo tratamiento.

Se estableció un estudio retrospectivo, observacional y descriptivo durante enero a diciembre del año 2016. El universo escogido fueron pacientes atendidos en las áreas de emergencias, cuidados críticos y hospitalización del Hospital Abel Gilbert Pontón, la muestra fueron 109 pacientes que presentaron Cáncer Colorrectal incluidos 41 nuevos casos en el período de tiempo de estudio establecido.

Se determinó los principales factores de riesgo relacionados al desarrollo y presentación de Cáncer Colorrectal, se identificó las manifestaciones clínicas frecuentes que se presentaron durante el año 2016, se realizaron tablas de frecuencia, cuadros y diagramas cruzados para establecer correlaciones importantes con significancia estadística descriptiva que se mostrarán a continuación. 


\section{Cáncer colorrectal en el hospital Guayaquil Dr. Abel Gilbert Pontón en el 2016}

Vol. 2, núm. Esp., (2018)

Henry Leonardo Triviño Yépez; Betzi Tatiana Vidal Zamora; Oswaldo Juan Zambrano Chávez

Frecuencia de edad de los pacientes con cáncer colorrectal en el año 2016.

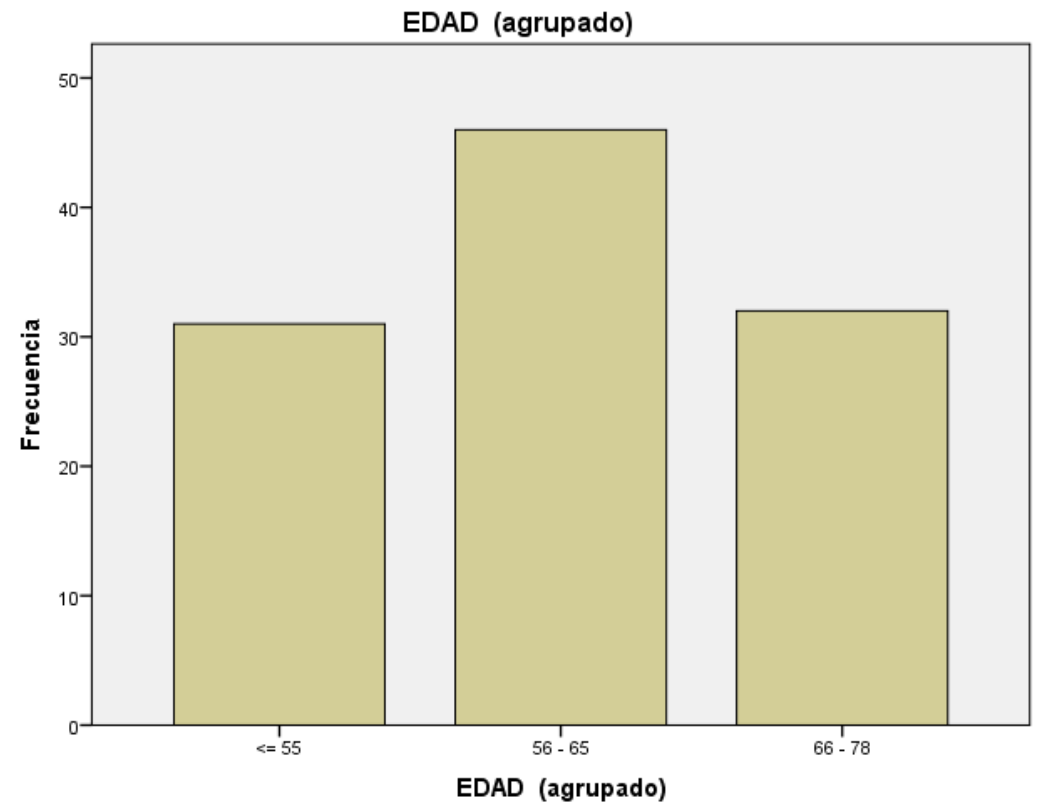

GRÁFICO 1. Distribución de los grupos de edad de pacientes con Cáncer Colorrectal en el 2016

Se muestra mayor frecuencia de cáncer colorrectal en pacientes entre edades de 56 a 65 años, de 109 pacientes 46 están en este rango correspondiendo un 42,2 \%; hay una frecuencia casi similar entre los grupos etarios de 45 a 55 años y 66 y 78 años correspondiendo a 28,4\% y $29,4 \%$ respectivamente. 


\section{Cáncer colorrectal en el hospital Guayaquil Dr. Abel Gilbert Pontón en el}

Vol. 2, núm. Esp., (2018)

Henry Leonardo Triviño Yépez; Betzi Tatiana Vidal Zamora; Oswaldo Juan Zambrano Chávez Género de pacientes con cáncer colorrectal en el 2016

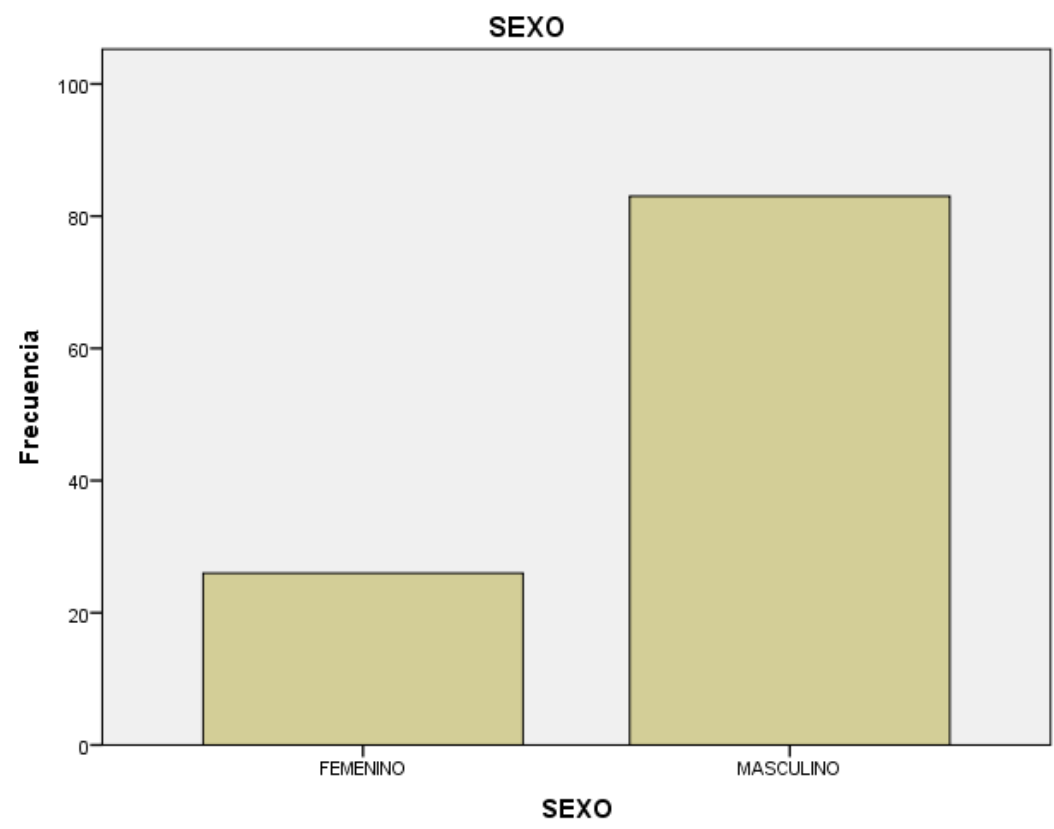

GRÁFICO 2. Distribución de género de pacientes con Cáncer Colorrectal en el 2016

Se demuestra mayor incidencia de pacientes masculinos que presentaron Cáncer de Colon durante el año 2016 , de nuestro total el 76,1\% fueron pacientes masculinos y el $23,9 \%$ fueron pacientes femeninas correlacionando nuestros resultados con los de la literatura, los varones presentan un mayor riesgo de desarrollar adenomas colonicos y Cáncer Colorrectal, así como una mayor mortalidad por éste. 


\section{Cáncer colorrectal en el hospital Guayaquil Dr. Abel Gilbert Pontón en el 2016}

Vol. 2, núm. Esp., (2018)

Henry Leonardo Triviño Yépez; Betzi Tatiana Vidal Zamora; Oswaldo Juan Zambrano Chávez

Frecuencia de manifestaciones clínicas de pacientes con cáncer colorrectal en el año 2016

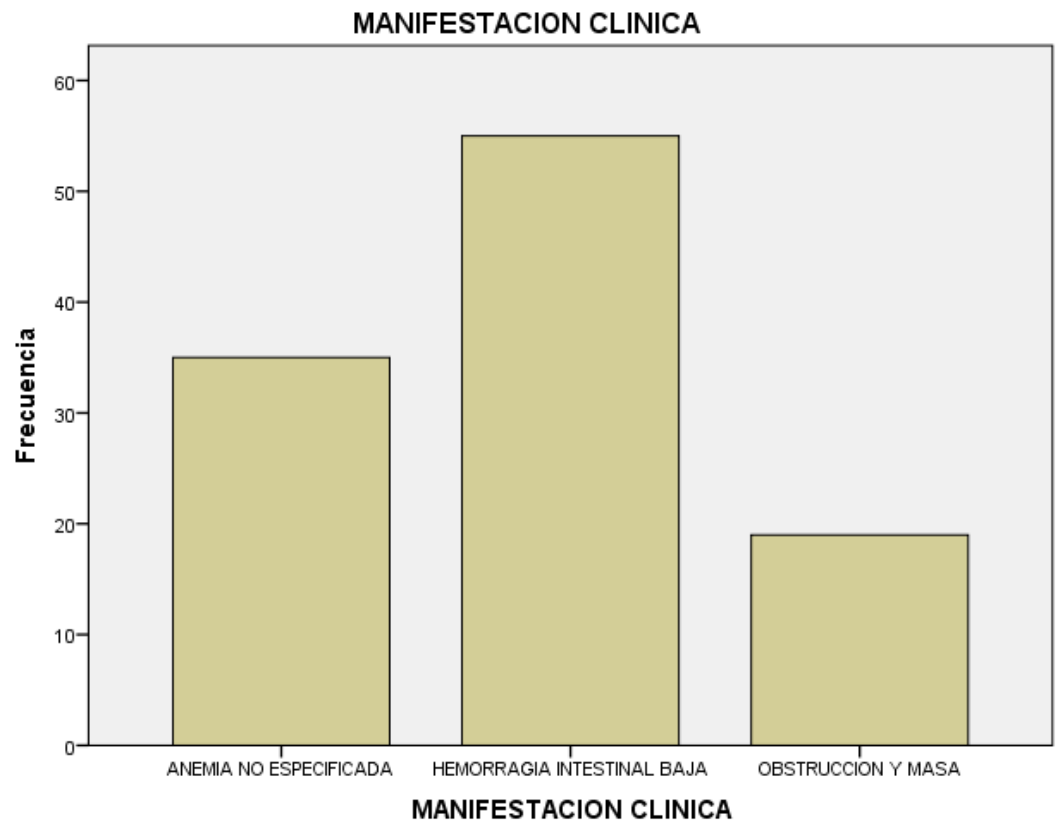

GRÁFICO 5. Representación de manifestaciones clínicas de los pacientes con Cáncer Colorrectal en el año 2016

La principal manifestación clínica del cáncer de colon es la hemorragia digestiva baja se encontró en nuestro estudio que el 50,5 \% del total presentaron este tipo de manifestación, el $32,1 \%$ presento un síndrome constitucional y asténico por anemia severa de tipo inespecífico y el 17,4\% presentaron obstrucción intestinal. 


\section{Cáncer colorrectal en el hospital Guayaquil Dr. Abel Gilbert Pontón en el}

Vol. 2, núm. Esp., (2018)

Henry Leonardo Triviño Yépez; Betzi Tatiana Vidal Zamora; Oswaldo Juan Zambrano Chávez Tipo de cáncer de colon en el año 2016. Diagnóstico de colonoscopía

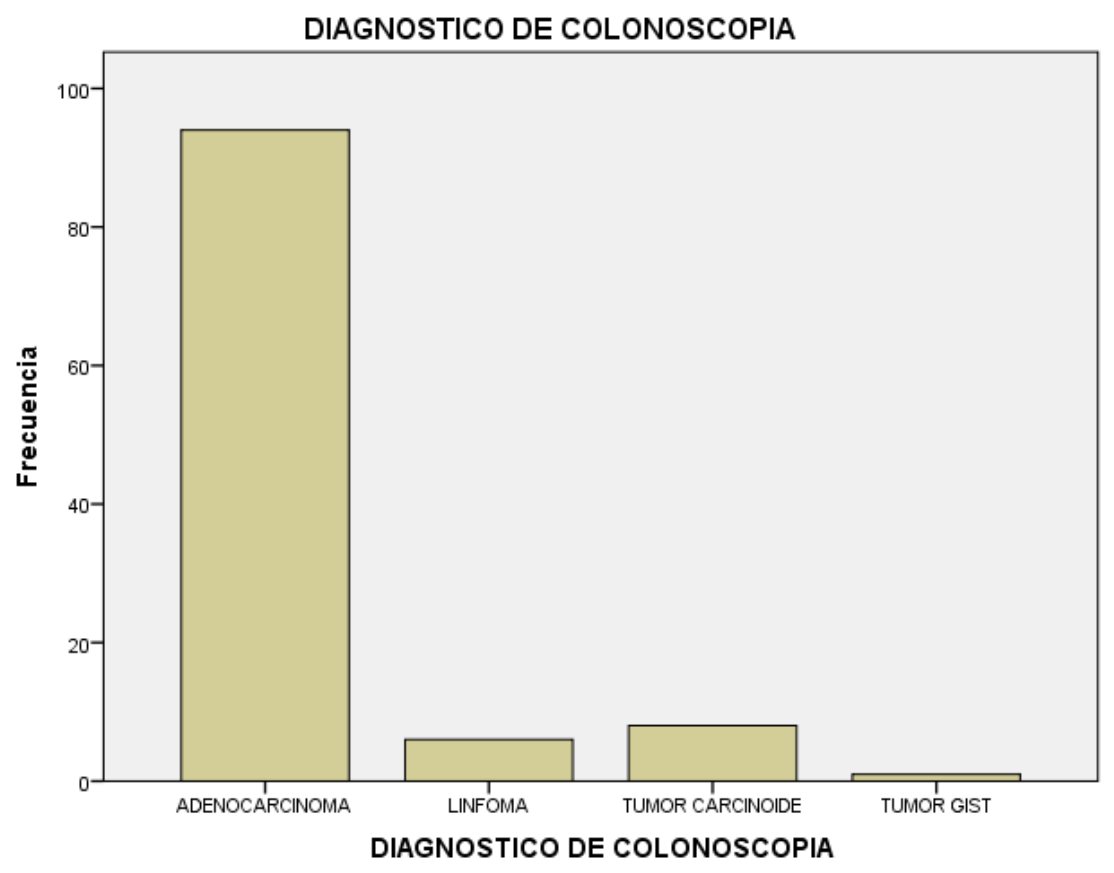

GRÁFICO 3. Distribución de diagnóstico por endoscopia y biopsia del ca de colon en el 2016

La interpretación del tipo de Cáncer Colorrectal más frecuente fue el ADENOCARCINOMA, entre los 109 pacientes del estudio 94 son de tipo adenocarcinoma, es decir el $86,2 \%$, mientras que el tumor carcinoide, linfoma y los tumores neuronales asociados a estroma intestinal (GIST) representaron en conjunto al $13.7 \%$. 


\section{Cáncer colorrectal en el hospital Guayaquil Dr. Abel Gilbert Pontón en el 2016}

Vol. 2, núm. Esp., (2018)

Henry Leonardo Triviño Yépez; Betzi Tatiana Vidal Zamora; Oswaldo Juan Zambrano Chávez

Frecuencia en localización del cáncer colorrectal en el año 2016

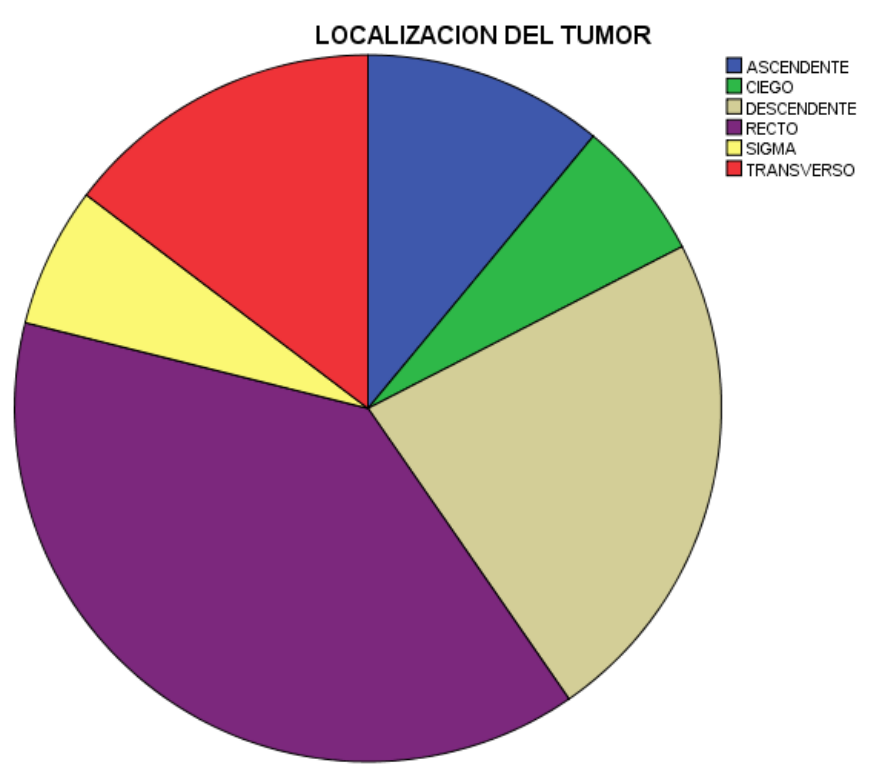

\section{GRÁFICO 4. Distribución y localización del cáncer de colon 2016}

La interpretación de la localización determinada por endoscopia baja la más frecuente fue en recto que representó $38,5 \%$, el colon descendente fue la segunda en frecuencia de localización que representa el 22,9 \% el cáncer de colon ascendente y ciego no es tan frecuente según la literatura, y en nuestro estudio se constata con frecuencias de 11\% de localización ascendente, ciego $6,4 \%$ y sigma $6,4 \%$. 


\section{Cáncer colorrectal en el hospital Guayaquil Dr. Abel Gilbert Pontón en el}

Vol. 2, núm. Esp., (2018)

Henry Leonardo Triviño Yépez; Betzi Tatiana Vidal Zamora; Oswaldo Juan Zambrano Chávez Tabla de frecuencia de estadio de cáncer colorrectal estadio del tumor

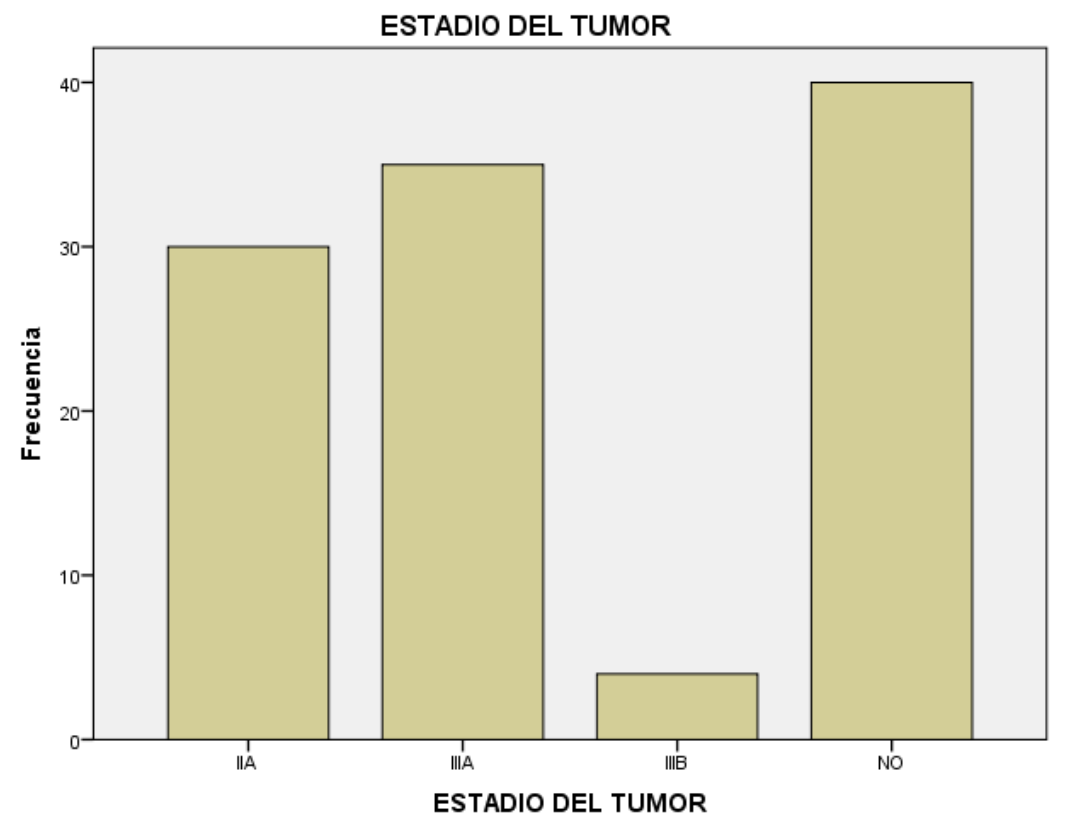

\section{GRÁFICO 4. Distribución según etapas clínicas del Cáncer Colorrectal en el 2016}

Se deduce que de 109 pacientes el $36,7 \%$ no ha sido estadificado aun, posterior a esto encontramos que el estadio IIIA representó $32,1 \%$, el estadio IIA en un 27,5\% y el estadio IIIB en un menor porcentaje de $3,7 \%$. 


\section{Cáncer colorrectal en el hospital Guayaquil Dr. Abel Gilbert Pontón en el}

2016

Vol. 2, núm. Esp., (2018)

Henry Leonardo Triviño Yépez; Betzi Tatiana Vidal Zamora; Oswaldo Juan Zambrano Chávez

Casos de cáncer colorrectal diagnosticados en el año 2016

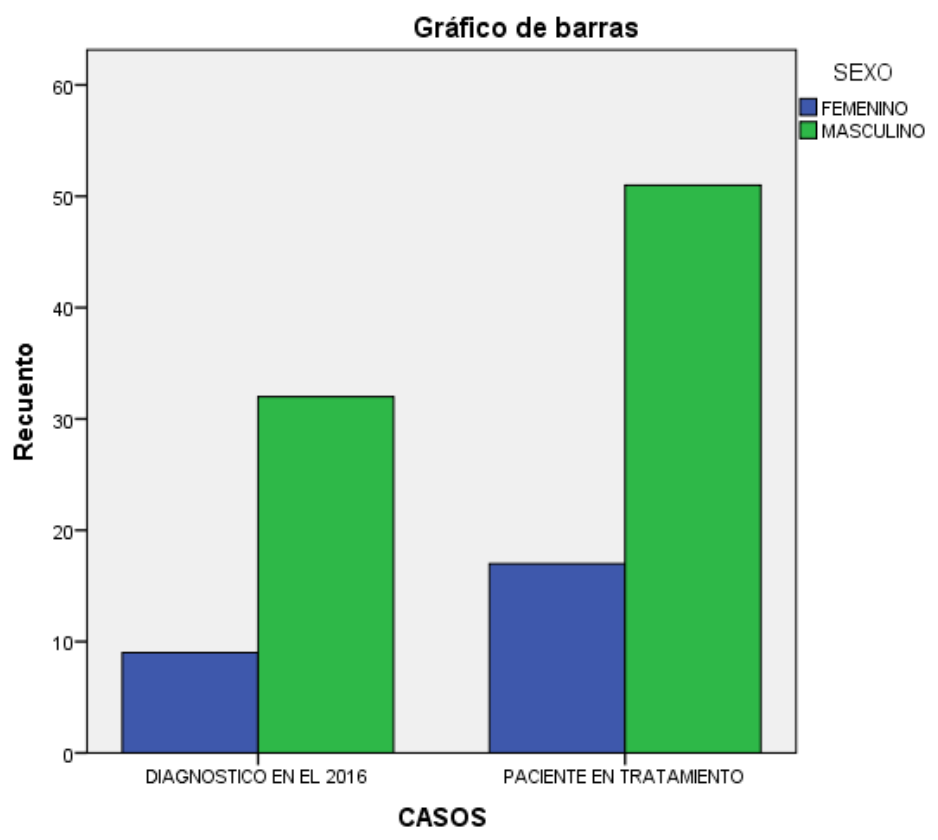

GRÁFICO 5. Distribución de los casos diagnosticados en el 2016 y otros años en un total de 109

Este grafico representa que, entre 109 pacientes, 41 fueron diagnosticados durante el 2016 correspondiendo al 37,6\% mientras que el 62,4\% faltante, es decir 68 pacientes se les había detectado cáncer de colon años anteriores. 


\section{Cáncer colorrectal en el hospital Guayaquil Dr. Abel Gilbert Pontón en el}

Vol. 2, núm. Esp., (2018)

Henry Leonardo Triviño Yépez; Betzi Tatiana Vidal Zamora; Oswaldo Juan Zambrano Chávez Correlación entre género y tipo de cáncer colorrectal en el año 2016

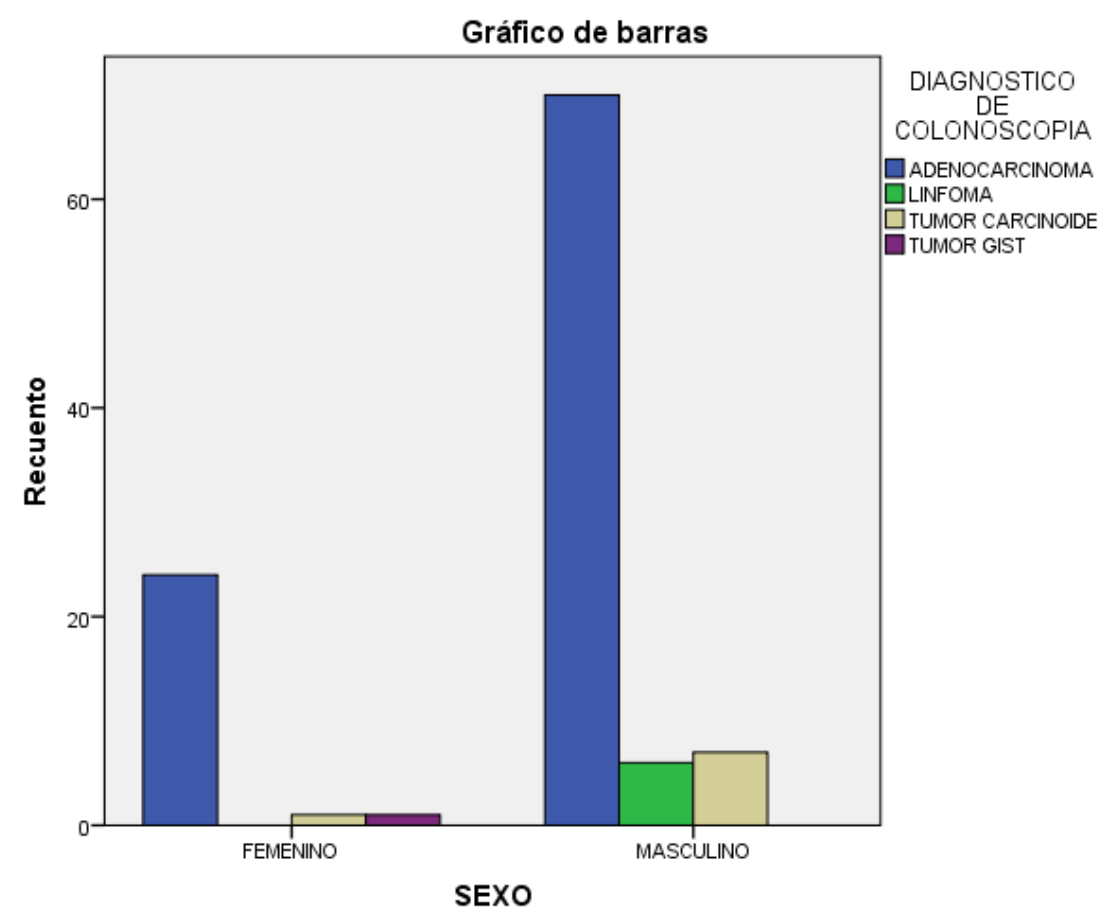

GRÁFICO 6. Diagrama de barras del tipo de cáncer colorrectal y la distribución en género, aก̃o 2016

El adenocarcinoma es el tumor más frecuente de localización en el intestino grueso, en estos diagramas podemos distinguir que su incidencia es mayor en varones, en este caso equivale a un $64,2 \%$, en pacientes femeninas se presentó una incidencia del $22 \%$. 


\section{Cáncer colorrectal en el hospital Guayaquil Dr. Abel Gilbert Pontón en el 2016}

Vol. 2, núm. Esp., (2018)

Henry Leonardo Triviño Yépez; Betzi Tatiana Vidal Zamora; Oswaldo Juan Zambrano Chávez

Correlación de tipo de cáncer colorrectal y su localización

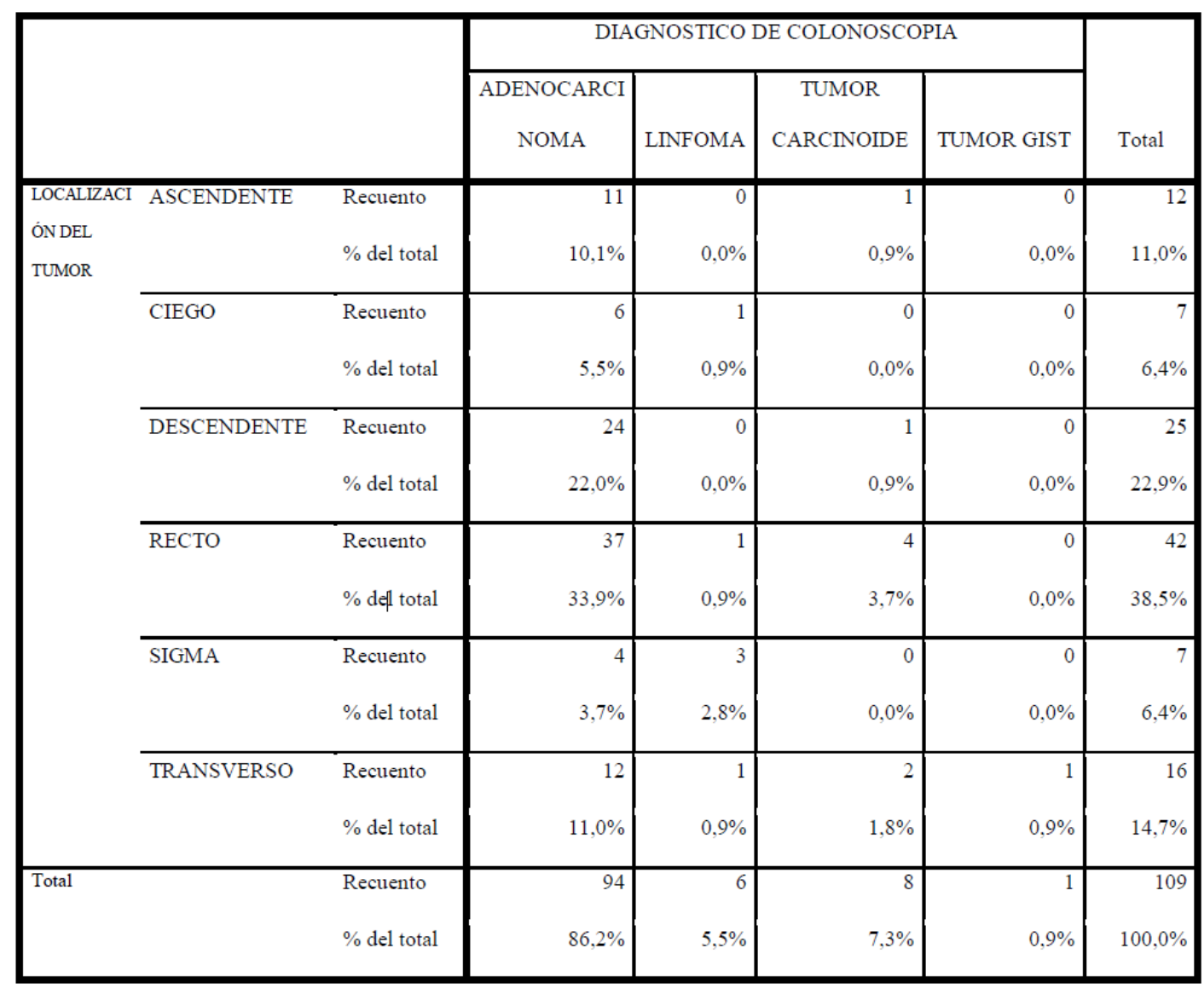

TABLA 1. Correlación del Cáncer Colorrectal y su localización 2016

El adenocarcinoma de colon fue el tipo de tumor con más incidencia en nuestro grupo de pacientes para este estudio, representó el 86,2\% de los casos, correlacionando este resultado con su localización se observó que su localización más frecuente fue en el colon descendente y en el recto, representado con el $33,9 \%$ y el $22 \%$ del total. 


\section{Cáncer colorrectal en el hospital Guayaquil Dr. Abel Gilbert Pontón en el}

Vol. 2, núm. Esp., (2018)

Henry Leonardo Triviño Yépez; Betzi Tatiana Vidal Zamora; Oswaldo Juan Zambrano Chávez

\section{Discusión.}

El cáncer de colon y recto (CCR) es el más frecuente en la actualidad, y presenta una mayor incidencia en varones. El 90\% de los casos de CCR se diagnostican por encima de los 50 años. Los principales factores de riesgo son los antecedentes familiares (CCR familiar, síndrome de Lynch o poliposis adenomatosa familiar [PAF]) y personales de CCR, los adenomas colónicos y la enfermedad inflamatoria intestinal (EII) colónica.

En la actualidad, se sitúa como el cáncer más frecuente en el global de la población, siendo el tercer tipo de cáncer más frecuente en varones, tras el de pulmón y próstata, y el segundo en mujeres tras el de mama. En cuanto a la distribución entre sexos, existe una mayor incidencia en varones que en mujeres.

Los resultados que demostró el estudio fueron relevantes demostrando mayor incidencia de cáncer de colon en pacientes entre edades de 56 a 65 años correspondiendo al 42,2\% seguido de los pacientes entre 45 a 55 años y 66 a 78 años que representaron el $28,4 \%$ y $29,4 \%$ respectivamente. Se plantea que nos encontramos en una base estándar comparada con los datos determinados en el estudio que realizó la Sociedad de Lucha Contra el Cáncer ecuatoriana.

Se determina en este estudio que entre 109 pacientes que fueron tratados e ingresados en el Hospital Abel Gilbert pontón, 41 fueron diagnosticados durante el 2016 correspondiendo al 37,6\% mientras que el 64,2\% faltante es decir 68 pacientes se les había diagnosticado cáncer de colon y recto en años anteriores y que asistieron al hospital por complicaciones del mismo. 


\section{Cáncer colorrectal en el hospital Guayaquil Dr. Abel Gilbert Pontón en el 2016}

Vol. 2, núm. Esp., (2018)

Henry Leonardo Triviño Yépez; Betzi Tatiana Vidal Zamora; Oswaldo Juan Zambrano Chávez

Según el tipo histológico de Cáncer Colorrectal, se determinó que sin importar edad de presentación, el adenocarcinoma fue el que mayor incidencia representó. Entre los 109 pacientes del estudio, 94 son de tipo adenocarcinoma, es decir el 86,2\%, mientras que el tumor carcinoide, linfoma y los tumores neuronales asociados a estroma intestinal (GIST) representaron en conjunto el $13.7 \%$

Se sabe que la mayoría de los casos de CCR tienen su origen en adenomas colónicos, por lo que la hipótesis de la secuencia adenoma-carcinoma es la más aceptada en la carcinogénesis colónica.

Existen numerosos datos que avalan esta hipótesis, como la presencia de carcinomas precoces sobre pólipos adenomatosos de gran tamaño, la misma distribución anatómica de adenomas y carcinomas colorrectales, la edad media de presentación de los adenomas 10-15 años antes de la aparición de adenocarcinoma tanto en casos esporádicos como familiares, el desarrollo de adenomas previos al carcinoma en modelos animales de carcinogénesis colónica y la reducción de la incidencia de CCR en poblaciones sujetas a seguimiento endoscópico y extirpación de los adenomas colorrectales.

En nuestro estudio se determinó que en nuestros pacientes la principal manifestación clínica del cáncer de colon fue hemorragia digestiva baja, se encontró que el 50,5 \% del total presenta este tipo de manifestación, el 32,1\% presentó un síndrome constitucional y asténico por anemia severa de tipo inespecífico y el 17,4\% presentó obstrucción intestinal. 


\section{Cáncer colorrectal en el hospital Guayaquil Dr. Abel Gilbert Pontón en el}

Vol. 2, núm. Esp., (2018)

Henry Leonardo Triviño Yépez; Betzi Tatiana Vidal Zamora; Oswaldo Juan Zambrano Chávez

\section{Conclusiones.}

Las conclusiones que a continuación se detallan se han elaborado en base a los objetivos alcanzados:

- La mayor incidencia de casos de Cáncer Colorrectal se diagnosticó por encima de los 56 años de edad.

- Se determinó que entre 109 pacientes que fueron tratados e ingresados en el Hospital Abel Gilbert Pontón, 41 fueron diagnosticados durante el año 2016 correspondiendo al $37,6 \%$ de la muestra.

- El tipo de tumor más frecuente fue el ADENOCARCINOMA COLORRECTAL.

- Se Observó una mayor incidencia de Cáncer Colorrectal en pacientes masculinos con el $76,1 \%$ del total.

- La localización del Cáncer Colorrectal tuvo mayor frecuencia en el Recto 38,5\% y en el colon descendente con el 22,9\% del total.

- El estadio clínico que presentó mayor reiteración en las pacientes con las que se diagnostican neoplasia de colón, fue el IIA y IIIA, sin embargo, el 36,7\% de neoplasias aún no han sido clasificadas en nuestro estudio.

- La manifestación clínica más frecuente fue la hemorragia digestiva baja que representó el 50,5\% de los pacientes que fueron ingresados por Cáncer Colorrectal. 


\section{Cáncer colorrectal en el hospital Guayaquil Dr. Abel Gilbert Pontón en el 2016}

Vol. 2, núm. Esp., (2018)

Henry Leonardo Triviño Yépez; Betzi Tatiana Vidal Zamora; Oswaldo Juan Zambrano Chávez

\section{Bibliografía.}

Abraldes. (2013). Tumores de intestino grueso. Medicine, 16-17.

Boardman. (2011). Cáncer colorrectal de aparición temprana en pacientes con predisposición genetica conocida. Medicine (Baltimor), 78-79.

Carolyn. (2015). Patología y los determinantes pronósticos de cáncer colorrectal. Uptodate, 3436.

Carraro. (2011). La obstruccion de cancer de colon: el fracaso y los patrones de supervivencia durante el seguimiento de diez años despues de la cirugia curativa en una sola etapa. Jama, 202-204.

Doubeni. (2012). El estatus socioeconómico y el riesgo de cáncer colorrectal: un análisis de más de medio millón de adultos en los institutos nacionales de Salud. Cancer 2012, 210-211.

Finlay. (2016). Presentación Clínica, diagnóstico y estatificación del cáncer colorrectal. JAMA, 80-83.

Garcia. (2015). Patología obstructiva del intestino grueso. Medicine, 101-103.

Girón. (2012). Protocolo diagnóstico de la lesión ulcerosa del recto. Protocolo de practica asistencial, 30-31.

Jemal. (2011). Estadísticas del cáncer a nivel mundial. CA Cancer J Clin, 101-114.

Rivero. (2013). Polipos y Poliposis colica. Medicine, 30-34.

Rodriguez-Bigas. (2016). Descripción General de la gestión de cáncer de colon primario. JAMA, 67-69.

Ryerson. (2016). Informe Anual a la Nación sobre el Estado del Cáncer. Jama 2016, 70-73.

Sanchez. (2012). Patología inflamatoria de recto y ano. Medicine, 23-27.

Siegel. (2016 ). Estadísticas del cáncer de colon . J clinic .

SOLCA. (2010). Centro de control de tumores en la ciudad de Guayaquil. Solca, 52-60.

Stoffel. (2015). Síndromes Hereditarios del cáncer colorrectal. Sociedad Europea de Guías Practica Clínica Oncológica, 23-25.

Teran. (2012). Cáncer de colon y recto. Medicine, 300-302.

Torre, D. 1. (2015). Estadísticas globales del cáncer. Cáncer J Clinic, 77-78. 


\section{Cáncer colorrectal en el hospital Guayaquil Dr. Abel Gilbert Pontón en el}

Vol. 2, núm. Esp., (2018)

Henry Leonardo Triviño Yépez; Betzi Tatiana Vidal Zamora; Oswaldo Juan Zambrano Chávez

Vasquéz. (2012). Protocolo diagnóstico y terapéutico del síndrome de obstrucción intestinal. Protocolos de Practica Asistencial, 17-19.

Weiss. (2011). Mortalidad por la etapa de derecha contra el cáncer de coon del lado izquierdo. $J$ Clinic Oncol, 34-45. 